\title{
VALORES HEMATOLÓGICOS Y DE BIOQUÍMICA SÉRICA DEL MONO CHORO COMÚN (Lagothrix lagotricha) CRIADO EN SEMICAUTIVERIO EN EL TRÓPICO PERUANO
}

\section{Haematological and Biochemestry Serum Values of Common Wolly MONKEY (Lagothrix lagotricha) REARED IN SEMI-CAPTIVITY IN THE Peruvian Tropics}

\author{
Katherine Rodríguez H. ${ }^{1}$, Miluska Navarrete Z. ${ }^{1,2}$, Olga Lí E. ${ }^{2}$, Luis Hoyos S. ${ }^{2}$, \\ Roberto Dávila F. ${ }^{3}$, Boris Lira M. ${ }^{4}$, Milagros Ramos M. ${ }^{5}$
}

\section{ResUMen}

El objetivo de este estudio fue determinar los valores hematológicos y de bioquímica sérica de una población de monos choros (Lagothrix lagotricha) que se encontraban en semicautiverio en el Centro de Rescate y Rehabilitación Ikamaperu, en Lagunas, Loreto. Se trabajó con 40 monos, 12 machos y 28 hembras entre juveniles, sub-adultos y adultos en aparente buen estado de salud. Los valores hematológicos fueron similares, los niveles de triglicéridos y de AST estuvieron elevados y los de proteínas totales y ALP estuvieron bajos en relación a estudios realizados en zoológicos. El grupo etario influenció los niveles de eritrocitos, proteínas totales, bilirrubina total, colesterol y fosfatasa alcalina; asimismo, no hubo diferencias por efecto del sexo en la serie eritrocítica, conteo de plaquetas y bioquímica sérica, con excepción del número de monocitos.

Palabras clave: mono choro común, Lagothrix lagotricha, hematología, bioquímica sérica, semicautiverio

\section{Abstract}

The objective of this study was to determine the haematological and serum biochemistry values in a population of woolly monkeys choros (Lagothrix lagotricha) reared in semi-captivity at the Centro de Rescate y Rehabilitación Ikamaperu in Lagunas, Loreto, Peru. Forty primates, 12 males and 28 females, which included juveniles, sub-

\footnotetext{
${ }^{1}$ Laboratorio de Anatomía Animal y Fauna Silvestre, ${ }^{2}$ Laboratorio de Patología Clínica y Biología Molecular, ${ }^{3}$ Clínica de Animales Menores, ${ }^{4}$ Laboratorio de Fisiología Animal, Facultad de Medicina Veterinaria, Universidad Nacional Mayor de San Marcos, Lima, Perú

${ }^{5}$ Centro de Rescate y Rehabilitación de Primates Ikamaperu, Lagunas, Loreto, Perú

${ }^{6}$ E-mail: miluskabeatriz@gmail.com
} 
adults and adults in apparent good health conditions were sampled. The haematological values were similar, triglyceride and AST values were elevated, and total protein and ALP values were low as compared to other studies in zoos. The age group influenced erythrocyte counts, total protein, total bilirubin, cholesterol and alkaline phosphatase. No sex effect was found on erythrocytes, leukocytes and platelet counts and in serum chemistry values, except for the number of monocytes.

Key words: common woolly monkey, Lagothrix lagotricha, hematology, serum biochemistry, semi-captivity

\section{INTRODUCCIÓN}

El mono choro común (Lagothrix lagotricha) es una de las 39 especies de primates que existe en el Perú (Pacheco et al., 2009), siendo su densidad poblacional bastante baja, dada la alta presión de caza y deforestación (Aquino y Encarnación, 1994).

La reglamentación para la conservación de la fauna amenazada en el Perú está bajo el amparo del Decreto Supremo $N^{\circ} 158-77$ AG, que define a las categorías de amenaza como especies En Vías de Extinción (E), Vulnerable (V), Rara (R) e Indeterminada (I). El mono choro común se encuentra en situación vulnerable y en el apéndice II del CITES (Wallace et al., 2008); así también se les clasifica como CR (seriamente amenazada) en la lista roja de la IUCN (Unión Internacional para la Conservación de la Naturaleza) (Jaramillo y Pérez, 2007). Las especies en situación vulnerable son aquellas que por exceso de caza o por destrucción de hábitat, son susceptibles de pasar a la situación de especies en vías de extinción. Estas especies necesitan apoyo de programas de conservación ex situ, como centros de rehabilitación y rescate. Una de las finalidades de estos programas es recuperar sanitaria, física, psíquica y conductualmente a un animal silvestre con la finalidad de ser liberado (Aprile, 1994), y para ello es indispensable, entre otros factores, un control sanitario previo (Evans, 1985).
Para este efecto, se requiere disponer de valores referenciales clínicos y de laboratorio para cada especie y dentro de las condiciones medioambientales de crianza para facilitar el control sanitario y servir como ayuda diagnóstica. Los valores de hematología y bioquímica sérica reportados en primates usualmente se encuentran dentro de los límites normales de mamíferos (Wallach y Boever, 1983); no obstante, los datos reportados de valores hematológicos en primates, incluyendo el mono choro son muy limitados (Wallach y Boever, 1983; ISIS, 2002; Jaramillo y Pérez, 2007; Ange-van Heugten, 2008).

El objetivo del estudio fue determinar los valores hematológicos y de bioquímica sérica en una población de monos choros que se encontraban en semicautiverio en el trópico peruano.

\section{Materiales y Métodos}

Se recolectaron muestras de sangre de monos choro común del Centro de Rescate y Rehabilitación Ikamaperu, ubicado en la comunidad de Lagunas, departamento de Loreto, Perú, durante febrero de 2011.

Se utilizó toda la población de monos choro común, consistente en 40 animales, 12 machos y 28 hembras, aparentemente sanos, agrupados según edad en 21 juveniles (hasta los cuatro años), 16 sub-adultos (de cinco a nueve años) y 3 adultos (de 10 o más años). 
Toda la población de monos choro común se encontraban bajo las mismas condiciones de manejo y alojamiento en semicautiverio y de alimentación (principalmente frutas de la zona como sandía, piña, zapote, guabas, plátano, papaya; así como camote, zapallo y un complemento proteico).

Los animales estuvieron en ayuno el día de la toma de muestra. Fueron anestesiados con ketamina (10 mg/kg, vía intramuscular) aunque algunos individuos necesitaron, además, un tranquilizante (diazepam, $10 \mathrm{mg} / \mathrm{kg}$, vía oral) para lograr la recumbencia.

Los monos choros fueron identificados y pesados. Se tomaron las constantes fisiológicas de temperatura, frecuencia cardiaca y frecuencia respiratoria, y luego se procedió a la toma de la muestra de sangre por punción de la vena femoral usando agujas de calibre $21 \mathrm{G}$ x $1 "$.

Para las pruebas hematológicas se recolectó la sangre $(3 \mathrm{ml})$ en tubos estériles al vacío con anticoagulante EDTA. Así mismo, se hicieron frotis sanguíneos en láminas portaobjetos en el momento de la toma de muestra. Para las pruebas de bioquímica sanguínea, se recolectó $4 \mathrm{ml}$ de sangre en tubos sin anticoagulante. El suero fue obtenido luego de retraer el coágulo y, además, se centrifugó a $3000 \mathrm{rpm}$ durante 8 minutos. Todas las muestras fueron rotuladas y mantenidas en refrigeración hasta su traslado al Laboratorio de Patología Clínica de la Facultad de Medicina Veterinaria, Universidad Nacional Mayor de San Marcos, en Lima.

Para la determinación de la serie eritrocítica, leucocítica y plaquetaria se usó el procedimiento descrito por Kraft y Schillinger (1998). Se determinó el volumen del paquete celular o hematocrito (\%) por el método del microhematocrito, la hemoglobina $(\mathrm{g} / \mathrm{dl})$ por el método de la cianometahemoglobina, y para el recuento de eritrocitos o glóbulos rojos (x 10\%/ $\mu$ l) se utilizó la cámara de Neubauer y un microscopio de luz a 400X.
Los índices eritrocíticos definieron el tamaño y el contenido de hemoglobina del eritrocito. Se determinó el:

- Volumen Corpuscular Medio (VCM) en fentolitros (fl): (hematocrito x 10$) / \mathrm{N} .^{\circ}$ de eritrocitos

- Hemoglobina Corpuscular Media (HCM) en picogramos (pg): (hemoglobina x 10)/ $\mathrm{N} .{ }^{\circ}$ de eritrocitos

- Concentración de Hemoglobina Corpuscular Media (CHCM) en gramos por decilitros (g/dl): (hemoglobina x 10)/ hematocrito

Para el recuento de leucocitos o glóbulos blancos (x $\left.10^{3} / \mu \mathrm{l}\right)$ se utilizó la cámara de Neubauer y un microscopio de luz a 100X. Para el recuento diferencial de leucocitos (\%), se emplearon láminas portaobjeto teñidas con la tinción Wright. Para el recuento plaquetario o trombocítico (trombocitos $/ \mu \mathrm{l}$ ) se utilizaron las láminas preparadas para el recuento diferencial.

En la bioquímica sérica se determinaron los niveles de urea $(\mathrm{mg} / \mathrm{dl})$ y niveles de creatinina $(\mathrm{mg} / \mathrm{dl})$ mediante el método colorimétrico; niveles de proteínas totales $(\mathrm{g} / \mathrm{dl})$, niveles de albúmina $(\mathrm{g} / \mathrm{dl})$, niveles de alanino aminotransferasa o ALT (IU/L), niveles de aspartato amino transferasa o AST (IU/L), niveles de fosfatasa alcalina o ALP (IU/L), bilirrubina Total (BT) y bilirrubina directa $(B D)(\mathrm{mg} / \mathrm{dl})$, colesterol $(\mathrm{mg} / \mathrm{dl})$, triglicéridos $(\mathrm{mg} / \mathrm{dl})$, usando el método enzimático, empleando el procedimiento de Kraft y Schillinger (1998). Asimismo, se determinó los niveles de glucosa en sangre $(\mathrm{mg} / \mathrm{dl})$ usando tiras reactivas y glucómetro Bionime GM300.

Se utilizó estadística descriptiva, empleando la media aritmética como medida de tendencia central y la desviación estándar como medida de dispersión para el cálculo de los valores hematológicos y de bioquímica sérica. Asimismo, para hallar las diferencias entre los parámetros hematológicos y bioquímica sérica por efecto del sexo, se uti- 
lizó la prueba de T de Student para muestras independientes, y para el efecto de edad se utilizó el análisis de varianza (de una vía) completamente aleatorio (Daniel, 1996).

\section{Resultados y Discusión}

Los resultados de la serie eritrocítica y leucocítica se muestran en los cuadros 1,2 y 3. El número de eritrocitos fue dependiente del grupo etario (adultos: $7.16 \pm 0.37 \times 10^{6} / \mu \mathrm{l}$; sub-adultos: $5.68 \pm 1.06$ x 10\%/ 1 ; ;óvenes: 5.52 $\left.\pm 1.07 \times 10^{6} / \mu \mathrm{l}\right)(\mathrm{p}<0.05)$, resultado que ha sido descrito como significativo en un estudio desarrollado para otros primates de las familias Atelidae y Cebidae (Jaramillo y Pérez, 2007). Por otro lado, no hubo diferencia estadística en el conteo de eritrocitos ni en el conteo de plaquetas en relación al sexo.

En la serie leucocitaria, se encontró diferencia significativa $(\mathrm{p}<0.05)$ en relación al sexo en el conteo de monocitos $\left(0.028 \times 10^{3} / \mu 1\right.$ para hembras y $0.005 \times 10^{3} / \mu 1$ para machos); diferencia debida a que solo se encontró monocitos en un macho en comparación con ocho hembras que presentaron este tipo de células leucocitarias.

Los valores encontrados en la serie eritrocítica, serie leucocítica y conteo de plaquetas fueron similares a los valores reportados por Wallach y Boever (1983) e ISIS (2002).

Los resultados de los valores de la bioquímica sérica se muestran en el Cuadro 4 y por grupo etario en el Cuadro 5. Se encontró diferencia estadística en cuanto al grupo etario en los valores de proteínas totales, bilirrubina total, colesterol y fosfatasa alcalina, pero no se encontraron diferencias por efecto del sexo de los animales.

La bilirrubina total se incrementó y el colesterol disminuyó con la edad ( $\mathrm{p}<0.05)$. Por otro lado, la fosfatasa alcalina en el grupo juvenil tuvo niveles más altos que en el grupo de sub-adultos $(\mathrm{p}<0.05)$, posiblemente debido a que, en condiciones normales, los niveles de ALP se encuentran elevados durante los periodos de crecimiento rápido del hueso en animales jóvenes (Benjamín, 1991);

Cuadro 1. Valores de la serie eritrocítica y plaquetaria del mono choro común (Lagothrix lagotricha) criado en semicautiverio en Lagunas, Loreto

\begin{tabular}{lccc}
\hline Variable & Media & $\begin{array}{c}\text { Desviación } \\
\text { estándar }\end{array}$ & Valores extremos \\
\hline Eritrocitos $(\mathrm{x} \mathrm{10} / \mu \mathrm{l})$ & 5.71 & 1.10 & $4.4-8.4$ \\
Hemoglobina $(\mathrm{g} / \mathrm{dl})$ & 12.67 & 1.19 & $10.9-15.9$ \\
$\mathrm{Hematocrito} \%)$ & 40.93 & 5.85 & $30-60$ \\
$\mathrm{VCM}^{1}(\mathrm{fl})$ & 73.15 & 11.84 & $54.8-113.2$ \\
$\mathrm{HCM}^{2}(\mathrm{pg})$ & 22.76 & 3.51 & $17.0-32.5$ \\
$\mathrm{CHCM}^{3}(\mathrm{~g} / \mathrm{dl})$ & 31.19 & 2.05 & $26.5-37.3$ \\
$\mathrm{Plaquetas}(\mathrm{x} \mathrm{10} / \mu \mathrm{l})$ & 343.08 & 57.65 & $250-450$ \\
\hline${ }^{1}$ Volumen corpuscular medio & & & \\
${ }^{2}$ Hemoglobina corpuscular media & & &
\end{tabular}


Cuadro 2. Valores de la serie leucocítica relativa del mono choro común (Lagothrix lagotricha) criado en semic autiverio en Lagunas, Loreto $(\mathrm{n}=40)$

\begin{tabular}{lccc}
\hline Variable & Media & $\begin{array}{c}\text { Desviación } \\
\text { estándar }\end{array}$ & Valores extremos \\
\hline Leucocitos $(\mathrm{x} \mathrm{10} / \mu 1)$ & 8.33 & 1.64 & $5.7-12.7$ \\
Abastonados $(\%)$ & 0 & 0 & 0 \\
Neutrófilos (\%) & 53.03 & 13.25 & $29-85$ \\
Eosinófilos (\%) & 5.95 & 3.92 & $1-15$ \\
Basófilos (\%) & 0.98 & 1.14 & $0-6$ \\
Linfocitos (\%) & 39.78 & 12.55 & $10-69$ \\
Monocitos $(\%)$ & 0.28 & 0.55 & $0-2$ \\
\hline
\end{tabular}

Cuadro 3. Valores de la serie leucocítica absoluta del mono choro común (Lagothrix lagotricha) criado en semicautiverio en Lagunas, Loreto $(\mathrm{n}=40)$

\begin{tabular}{lccc}
\hline Variable & Media & $\begin{array}{c}\text { Desviación } \\
\text { estándar }\end{array}$ & Valores extremos \\
\hline Leucocitos $\left(\times 10^{3} / \mu \mathrm{l}\right)$ & 8.33 & 1.64 & $5.7-12.7$ \\
Abastonados $\left(\times 10^{3} / \mu \mathrm{l}\right)$ & 0 & 0 & 0 \\
Neutrófilos $\left(\times 10^{3} / \mu \mathrm{l}\right)$ & 4.42 & 1.39 & $2.2-7.6$ \\
Eosinófilos $\left(\mathrm{x} 10^{3} / \mu \mathrm{l}\right)$ & 0.5 & 0.36 & $0.07-1.65$ \\
Basófilos $\left(\mathrm{x} 10^{3} / \mu \mathrm{l}\right)$ & 0.08 & 0.1 & $0-0.5$ \\
Linfocitos $\left(\mathrm{x} 10^{3} / \mu \mathrm{l}\right)$ & 3.31 & 1.27 & $0.9-6.6$ \\
Monocitos $\left(\mathrm{x} 10^{3} / \mu \mathrm{l}\right)$ & 0.02 & 0.04 & $0-0.2$ \\
\hline
\end{tabular}

sin embargo, en el presente estudio, el grupo de adultos presentó los niveles más elevados. Se conoce que alteraciones que provocan una remodelación ósea en el adulto dan lugar a ligeras elevaciones que no llegan a duplicar los valores normales (Sodikoff, 1996).

Las diferencias por grupo etario para proteínas totales son similares a las halladas en el estudio de Ange-van Heugten (2008), aunque los valores de ese estudio fueron superiores en promedio $(7.1 \mathrm{~g} / \mathrm{dl})$. Asimismo, en ISIS (2002) se señalan promedios de $7.2 \mathrm{~g} / \mathrm{dl}$. Esta discrepancia podría deberse a la diferencia en el tipo de alimentación suministrada a los primates; sin embargo, los promedios de albúmina son similares, de allí que no hay un proceso de malnutrición o alteración similar en los individuos del presente estudio. 
Cuadro 4. Valores de bioquímica sérica en el mono choro común (Lagothrix lagotricha) criado en semicautiverio en Lagunas, Loreto $(n=40)$

\begin{tabular}{|c|c|c|c|}
\hline Variable & Media & $\begin{array}{l}\text { Desviación } \\
\text { estándar }\end{array}$ & Valores extremos \\
\hline Urea (mg/dl) & 26.97 & 3.68 & $20.9-41$ \\
\hline Creatinina (mg/dl) & 1.12 & 0.32 & $0.5-2.0$ \\
\hline Proteínas totales (g/dl) & 5.93 & 0.78 & $4.0-7.5$ \\
\hline Albúmina (g/dl) & 4.05 & 0.52 & $2.5-4.8$ \\
\hline $\operatorname{ALT}^{1}(\mathrm{UI} / \mathrm{L})$ & 36.43 & 3.14 & $29.8-43.9$ \\
\hline $\operatorname{AST}^{2}(\mathrm{UI} / \mathrm{L})$ & 108.26 & 21.88 & $15.5-147.2$ \\
\hline $\mathrm{BT}^{3}(\mathrm{mg} / \mathrm{dl})$ & 0.53 & 0.07 & $0.43-0.71$ \\
\hline $\mathrm{BD}^{4}(\mathrm{mg} / \mathrm{dl})$ & 0.27 & 0.05 & $0.1-0.4$ \\
\hline $\operatorname{ALP}^{5}(\mathrm{UI} / \mathrm{L})$ & 45.15 & 3.27 & $38.6-52.8$ \\
\hline Colesterol (mg/dl) & 131.1 & 27.14 & $14.4-193.7$ \\
\hline Triglicéridos (mg/dl) & 133.21 & 54.69 & $90.8-452.4$ \\
\hline $\operatorname{Glucosa}^{6}(\mathrm{mg} / \mathrm{dl})$ & 91.45 & 22.08 & $54-142$ \\
\hline
\end{tabular}

${ }^{1}$ Alanino amino transferasa

${ }^{2}$ Aspartato amino transferasa

${ }^{3}$ Bilirubina total

${ }_{5}^{4}$ Bilirubina directa

${ }^{5}$ Fosfatasa alcalina

${ }^{6}$ En base a muestras de 31 individuos

El valor promedio de AST (108.26 UI/ L) fue superior a otros reportes $(53 \mathrm{UI} / \mathrm{L}$ por ISIS, 2002; $80 \mathrm{UI} / \mathrm{L}$ por Ange-van Heugten, 2008), aunque con una gran variabilidad en estos promedios. La actividad enzimática de AST se podría encontrar elevada cuando hay un proceso de daño en músculo esquelético, tejido hepatocelular o en músculo cardíaco (Sodikoff, 1996). Antes de concluir que la actividad AST sérica elevada se debe a un daño hepático, se debe descartar la necrosis muscular midiendo la actividad de creatina quinasa (CK) (Latimer et al., 2005). Asimismo, se ha reportado problemas de salud en poblaciones de monos choros con respecto a trastornos de hígado y músculos, especialmente por la ganancia de peso en la preñez
(Ange-van Heugten et al., 2008). Investigaciones en seres humanos han demostrado que enzimas elevadas en el suero como ALT, AST y GGT, están asociadas con riesgo cardiovascular, incluyendo transtornos de hipertensión, como se observa en monos choros en cautiverio (Whitfield et al., 2002; Schindhelm et al., 2007).

El valor promedio hallado para ALP (45.15 UI/L) fue más bajo que los valores 231 y 139 UI/L reportados por ISIS (2002) y Ange-van Heugten (2008), respectivamente. Esto podría ser un indicativo de que los individuos del presente estudio no mostraban alteraciones que puedan inducir el aumento de la actividad enzimática de esta enzima; 
Cuadro 5. Valores de bioquímica sérica en el mono choro común (Lagothrix lagotricha) criado en semic autiverio en Lagunas, Loreto según grupo etario ${ }^{1}(n=40)$

\begin{tabular}{|c|c|c|c|c|}
\hline Variable & Grupo etario & Media & $\begin{array}{l}\text { Desviación } \\
\text { estándar }\end{array}$ & $\begin{array}{l}\text { Valores } \\
\text { extremos }\end{array}$ \\
\hline \multirow[t]{3}{*}{ Urea $(\mathrm{mg} / \mathrm{dl})$} & Juvenil $^{1}$ & 25.77 & 3.02 & $20.9-30.7$ \\
\hline & Sub adulto $^{2}$ & 28.54 & 4.05 & $22.9-41.0$ \\
\hline & Adulto ${ }^{3}$ & 27.03 & 3.90 & $23.1-30.9$ \\
\hline \multirow{3}{*}{ Creatinina (mg/dl) } & Juvenil & 1.01 & 0.28 & $0.46-1.42$ \\
\hline & Sub adulto & 1.25 & 0.31 & $0.78-2.03$ \\
\hline & Adulto & 1.20 & 3.90 & $0.76-1.66$ \\
\hline \multirow[t]{3}{*}{ Proteínas totales $(\mathrm{g} / \mathrm{dl}) *$} & Juvenil & 6.13 & 0.62 & $4.97-7.53$ \\
\hline & Sub adulto & 5.56 & 0.89 & $4.04-7.12$ \\
\hline & Adulto & 6.47 & $\begin{array}{l}0.09 \\
0.30\end{array}$ & $6.23-6.81$ \\
\hline \multirow[t]{3}{*}{ Albúmina (g/dl) } & Juvenil & 4.07 & 0.58 & $2.5-4.76$ \\
\hline & Sub adulto & 3.94 & 0.44 & $3.24-4.74$ \\
\hline & Adulto & 4.50 & 0.30 & $4.15-4.69$ \\
\hline \multirow[t]{3}{*}{ ALT (UI/L) } & Juvenil & 36.47 & 3.42 & $30.8-43.9$ \\
\hline & Sub adulto & $\begin{array}{l}30.41 \\
36.28\end{array}$ & $\begin{array}{l}5.42 \\
3.14\end{array}$ & $29.8-40.9$ \\
\hline & Adulto & 36.90 & $\begin{array}{l}5.14 \\
1.01\end{array}$ & $36-38$ \\
\hline \multirow[t]{3}{*}{ AST (UI/L) } & Juvenil & 111.59 & 16.56 & $90.5-147.2$ \\
\hline & Sub adulto & 106.26 & 28.45 & $15.5-147.2$ \\
\hline & Adulto & 95.67 & 11.57 & $83.7-106.8$ \\
\hline \multirow[t]{3}{*}{$\mathrm{BT}(\mathrm{mg} / \mathrm{dl}) *$} & Juvenil & 0.51 & 0.05 & $0.43-0.59$ \\
\hline & Sub adulto & 0.55 & 0.07 & $0.47-0.71$ \\
\hline & Adulto & 0.61 & 0.05 & $0.55-0.64$ \\
\hline \multirow[t]{3}{*}{$\mathrm{BD}(\mathrm{mg} / \mathrm{dl})$} & Juvenil & 0.28 & 0.04 & $0.19-0.38$ \\
\hline & Sub adulto & 0.26 & 0.06 & $0.14-0.37$ \\
\hline & Adulto & 0.29 & 0.03 & $0.26-0.31$ \\
\hline \multirow[t]{3}{*}{$\operatorname{ALP}(\mathrm{UI} / \mathrm{L})^{*}$} & Juvenil & 46.66 & 2.70 & $41.8-52.8$ \\
\hline & Sub adulto & 42.56 & 2.35 & $38.6-48.2$ \\
\hline & Adulto & 48.33 & 1.12 & \\
\hline \multirow[t]{3}{*}{ Colesterol $(\mathrm{mg} / \mathrm{dl})^{*}$} & Juvenil & 133.16 & 18.9 & $104.8-163.8$ \\
\hline & Sub adulto & 138.16 & 20.49 & $105.5-193.7$ \\
\hline & Adulto & 79.00 & 55.94 & $14.42-112.0$ \\
\hline \multirow[t]{3}{*}{ Triglicéridos (mg/dl) } & Juvenil & 122.57 & 19.28 & $90.8-164.1$ \\
\hline & Sub adulto & 142.46 & 84.19 & $95.8-452.4$ \\
\hline & Adulto & 123.30 & 17.45 & $103.6-136.8$ \\
\hline \multirow[t]{3}{*}{ Glucosa $(\mathrm{mg} / \mathrm{dl})^{2}$} & Juvenil & 91.33 & 22.87 & $57-142$ \\
\hline & Sub adulto & 90.17 & 24.03 & $54-142$ \\
\hline & Adulto & 98.67 & 4.04 & $94-101$ \\
\hline
\end{tabular}

1 Juvenil: hasta 4 años; Sub-adulto: entre 5 y 9 años; Adulto: 10 o más años

${ }^{2}$ En base a muestras de 31 individuos

* Diferencias estadísticas entre grupos etarios $(p<0.05)$ 
sin embargo, hay que considerar que ALP no es específica de un órgano y se debe tener en cuenta otros parámetros bioquímicos. Por ejemplo, niveles elevados de ALP junto a elevaciones de gamma glutamil transferasa (GGT) serían indicativos de colestasis (Latimer et al., 2005).

El valor promedio de triglicéridos hallado en el presente estudio $(133.21 \mathrm{mg} / \mathrm{dl})$ fue mayor que los valores de 42 y $53 \mathrm{mg} / \mathrm{dl} \mathrm{re}$ portados por ISIS (2002) y Ange-van Heugten (2008), respectivamente. Las dietas ricas en grasas pueden provocar una hiperlipemia post-prandial; asimismo, niveles elevados pueden ser debido a una hipertrigliceridemia por alteraciones hereditarias en el metabolismo de las lipoproteínas, hipertrigliceridemia postprandial, hipotiroidismo, diabetes mellitus, enfermedad hepática y síndrome nefrótico entre otros; sin embargo, se podrían descartar estas causas dado que los animales se encontraban en ayunas, no mostraban signos compatibles con hipotiroidismo ni niveles elevados de colesterol, ALT o presencia de anemia y los niveles de glucosa sérica se encontraban normales.

Los niveles de urea y creatinina se encontraban dentro de los valores reportados por otros estudios (Sodikoff, 1996; Latimer et al., 2005).

\section{Conclusiones}

- Los valores hematológicos obtenidos en el mono choro común (Lagothrix lagotricha) criados en semicautiverio en Lagunas, Loreto, son similares a otros estudios realizados en zoológicos.

- Los niveles de triglicéridos y de AST estuvieron elevados y los de proteínas totales y ALP estuvieron bajos en relación a otros estudios.

- El grupo etario influenció los niveles de eritrocitos, proteínas totales, bilirrubina total, colesterol y fosfatasa alcalina.
- No hubo diferencias por efecto del sexo en la serie eritrocítica, conteo de plaquetas y bioquímica sérica, con excepción del número de monocitos.

\section{Agradecimientos}

Los autores desean expresar su agradecimiento a las Médico Veterinario Marieke Hilarides R. y Blanca Acosta J. por su apoyo desinteresado en el muestreo, y a la asociación UPA (Unidos por los Animales) que colaboraron en el apoyo financiero de este trabajo. Asimismo, a Heléne y Carlos de Ikamaperu por su esfuerzo y el amor en la rehabilitación de primates, así como por la oportunidad de realizar este trabajo en sus instalaciones.

\section{Literatura Citada}

1. Ange-van Heugetn K. 2008. Nutritional analyses and intervention in the captive woolly monkey (Lagothrix lagotricha). PhD Thesis. The Netherlands: Wageningen University. $191 \mathrm{p}$.

2. Aprile G 1994. Plan de rehabilitación y reintroducción de monos carayá. Buenos Aires: Fundación Vida Silvestre Argentina. $24 \mathrm{p}$.

3. Aquino R, Encarnación F. 1994. Los primates del Perú. República Federal de Alemania: Ed Goltze. 107 p.

4. Benjamín M. 1991. Manual de patología clínica en veterinaria. México: Ed Limusa. 421 p.

5. Daniel W. 1996. Bioestadística para el análisis de las ciencias de la salud. $5^{a}$ ed. México: Ed Limusa. 667 p.

6. Dew JL. 2005. Foraging, food choice and food processing by sympatric ripefruit specialists: Lagothrix lagotricha poeppigii and Ateles belzebuth belzebuth. Int J Primatol 26: 11007-1135.

7. Evans AT. 1985. Introduction to wildlife rehabilitation. USA: National Wildlife Rehabilitators Association. 87 p. 
8. [ISIS] International Species Information System. 2002. Clinical pathology records report - ISIS. In house reference values mammals. [Internet]. Disponible en: http://www.isis.org

9. Jaramillo S, Pérez A. 2007. Parámetros hematológicos y química sanguínea en primates de las familias Atelidae y Cebidae del Centro de Atención y Valoración de Fauna Silvestre (CAV) y Zoológico de Santa Fe. Tesis de Médico Veterinario y Zootecnista. Medellín: Universidad CES. 58 p.

10. Kraft H, Schillinger D. 1998. Métodos de laboratorio clínico en medicina veterinaria de mamíferos domésticos. España: Acribia. 295 p.

11. Latimer KS, Mahaffey EA, Prasse $K W .2005$. Patología clínica veterinaria. $4^{\mathrm{a}}$ ed. España: Multimédica Ediciones Veterinarias. $550 \mathrm{p}$.

12. Pacheco V, Cadenillas $R$, Salas E, Tello C, Zeballos H. 2009. Diversidad y endemismo de los mamíferos del Perú. Rev Peru Biol 16(1): 5-32.

13. Schindhelm $R$, Dekker JM, Nijpels $G$ Bouter LM, Stehouwer CD, Heine
RJ, Diamant M. 2007. Alanine aminotransferase predicts coronary heart disease events. A ten-year follow-up of the Hoorn study. Atherosclerosis 191: 391-396.

14. Sodikoff C. 1996. Pruebas diagnósticas y de laboratorio en las enfermedades de pequeños animales. $2^{\mathrm{a}}$ ed. España: Ed Mosby. 435 p.

15. Wallace RB, Mittermeier RA, Cornejo F, Boubli JP. 2008. Ateles chamek. En: IUCN 2010. IUCN Red List of Threatened Species. Version 2010.4. [Internet]. Disponible en www.iucnredlist.org

16. Wallach JD, Boever WJ. 1983. Primates. In: Diseases of exotic animals: medical and surgical management. Philadelphia, USA: WB Saunders. p 3123.

17. Whitfield JB, Zhu G, Nestler JE, Health AC, Martin NG. 2002. Genetic covariation between serum gammaglutamyltransferase activity and cardiovascular risk factors. Clin Chem 48: 1426-1431. 